\title{
The key management of direct/external modulation semiconductor laser response systems for relative intensity noise control
}

\author{
Mahmoud M. A. Eid ${ }^{1}$, Ashraf S. Seliem², Ahmed Nabih Zaki Rashed ${ }^{3}$, Abd El-Naser A. Mohammed ${ }^{4}$, \\ Mohamed Yassin Ali ${ }^{5}$, and Shaimaa S. Abaza ${ }^{6}$ \\ ${ }^{1}$ Department of Electrical Engineering, College of Engineering, Taif University, Kingdom of Saudi Arabia \\ ${ }^{3,4}$ Electronics and Electrical Communications Engineering Department, Faculty of Electronic Engineering, Menoufia \\ University, Menouf, Egypt \\ ${ }^{2,5,6}$ Benha Faculty of Engineering, Benha University, Benha, Egypt
}

\begin{tabular}{l} 
Article Info \\
\hline Article history: \\
Received Jul 9, 2020 \\
Revised Sep 3, 2020 \\
Accepted Sep 18, 2020 \\
\hline Keywords: \\
Bias current \\
Direct modulation \\
External modulation \\
Inter-modulation distortion \\
Laser rate equations \\
Modulation peak current
\end{tabular}

\begin{abstract}
This study outlines the management of either direct or external modulation semiconductor laser systems for the key solution of bit rate up to $25 \mathrm{~Gb} / \mathrm{s}$ under relative intensity noise (RIN) control. The bias and modulation peak currents based laser rate equations are optimized to achieve max $\mathrm{Q}$ factor and min bit error rate (BER) using first proposed model and optical/electrical signal power, optical/electrical signal to noise ratio are also enhanced using second proposed model. The percentage enhancement ratio in max. Q-factor and min. BER using first proposed model ranges from $53.25 \%$ to $71.63 \%$ in compared to the previous model. In the same way, by using second proposed model, the electrical signal power at optical receiver is enhanced within the range of $48.66 \%$ to $68.88 \%$ in compared to the previous model. Optical signal/noise ratio (OSNR) after optical fiber cable (OFC), signal/noise ratio (SNR) after electrical filter are measured with using different electrical pulse generators and electrical modulators at the optimization stage.
\end{abstract}

This is an open access article under the CC BY-SA license.

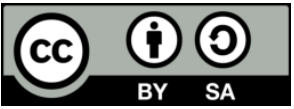

\section{Corresponding Author:}

Ahmed Nabih Zaki Rashed

Faculty of Electronic Engineering

Menoufia University

Menouf 32951, Egypt

E-mail: ahmed_733@yahoo.com

\section{INTRODUCTION}

They have analyzed the dynamics and noise of semiconductor laser communication systems for optical access television communication systems [1-7]. The typical frequency spectra of relative intensity noise are studied with modulation. When the modulation index is over $50 \%$, the modulated signal is clipped and the noise levels will increase. Relative intensity noise and modulation index equations are modeled mathematically. As well as small signal modulation transfer function is covered [8-17]. The modulated signal response of the laser is also characterized. The variations between modulation index and modulation channel frequency are reviewed. The relation between RIN frequency spectra and different modulation types are studied [1, 18-22]. They have presented the optimum parameters for the control of noise and distortion in semiconductor laser under analog modulation. The laser rate equations are modeled and integrated numerically. The relation between modulation index/channel, number of loaded channels and fiber length are reviewed mathematically [23-28]. The increase of fiber length limits the modulation index per channel and 
number of loaded channels. The total of carrier to noise ratio of semiconductor laser is estimated for fiber access communication television. Optimum noise and distortion of semiconductor laser are covered [2,3].

They have proposed the modulation semiconductor laser amplifier response by using optiwave simulation. The resonance frequency of laser is estimated for known threshold current, modulation peak current, and carrier lifetime based on laser rate equations [29-38]. The effect of increase modulation frequency above resonance at data rate of $10 \mathrm{~Gb} / \mathrm{s}$ is reviewed [4-6].

\section{MODEL DESCRIPTION AND RESEARCH METHOD}

The view of external laser system modulation is shown in Figure 1 (external modulation), where the user sequence generator is responsible for generating a series of bit sequence. The series of bits is represented by electrical pulse generators and then directed to low pass Gaussian filter to remove the ripple from the original signal. The filtered signal is routed to laser rate equations in order to convert the electrical signal to optical signal under the control of both modulation peak current and bias current. The Mach Zehnder modulators combine both the optical signal from laser and electrical signal from electrical pulse generator. The modulated signal is then directed to the optical fiber cable channel and then to optical attenuator which increases the signal losses level. The signal is then directed to optical receiver to convert the light signal to electrical signal.

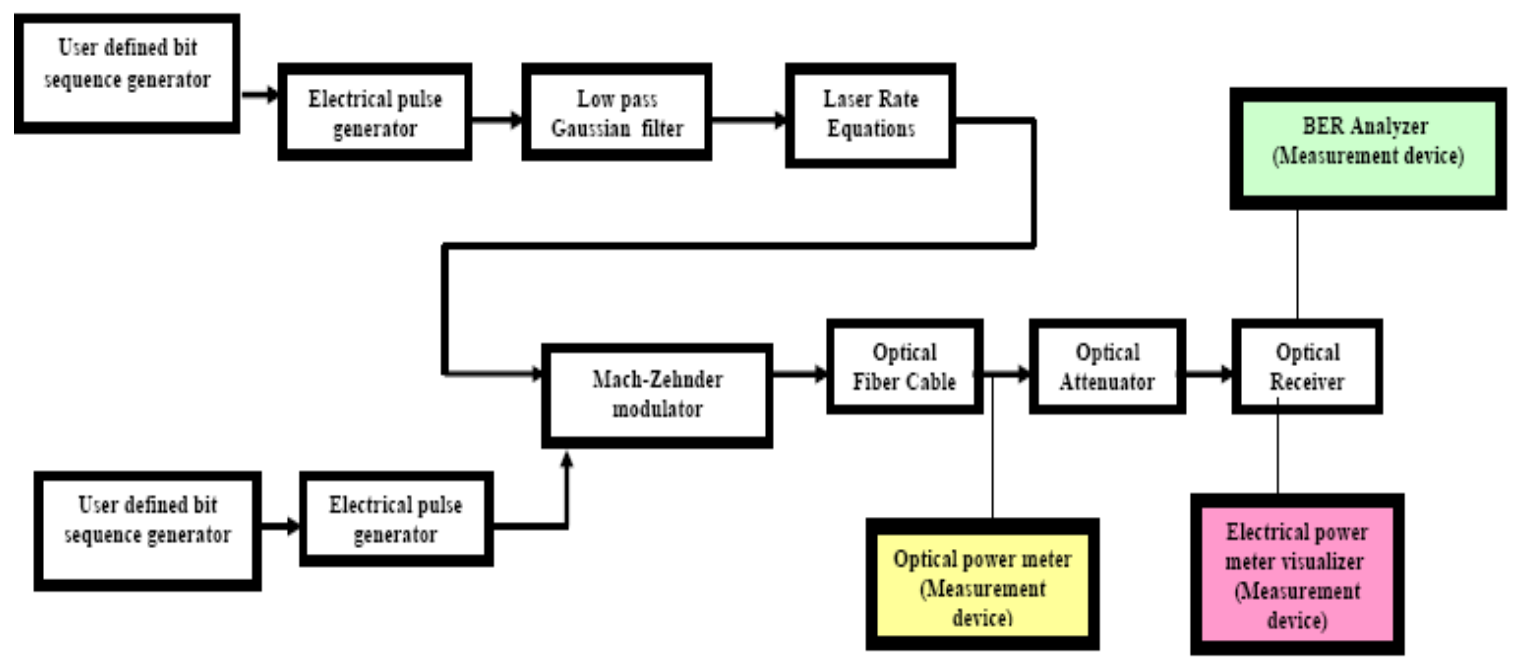

Figure 1. First proposed simulation model (external modulation)

Optical power meter measures the optical signal power through the optical fiber communication channel. Electrical power meter visualizer tests the electrical signal power at the receiving side. BER analyzer tests the max. Q-factor and minimum BER values at the optical receiver side. Figure 2 shows the direct modulation method of laser systems. The pseudo random bit sequence generator generates a random bit sequence and is directed to both electrical pulse generator and electrical modulators to be reshaped. The reshaped signal is directed to laser rate equations which converts the electrical signal to optical signal under the control of bias and maximum peak currents. The optical signal is then routed to optical fiber cable channel and then to PIN photo-detector which converts the optical signal to electrical signal. The converted signal is directed to band pass Gaussian filter in order to remove the unwanted signal (noise signal) for the original signal and then directed to $3 \mathrm{R}$ regenerator in order to achieve retiming, reshaping, regeneration for the filtered signal. WDM analyzer tests the optical signal to noise ratio through the optical fiber cable channel and noise power through optical fiber. Optical power meter tests the optical signal power at optical fiber cable channel. BER analyzer test the max. Q-factor and min BER values. Electrical carrier analyzer after band pass Gaussian filter measures the signal to noise ratio (signal power/noise power). Oscilloscope visulaizer after both PIN photodetector and band pass Gaussian filter tests maximum signal amplitude variations against bit period time variations. As well as electrical power meter visulaizer after PIN photodetector measures electrical signal power. 


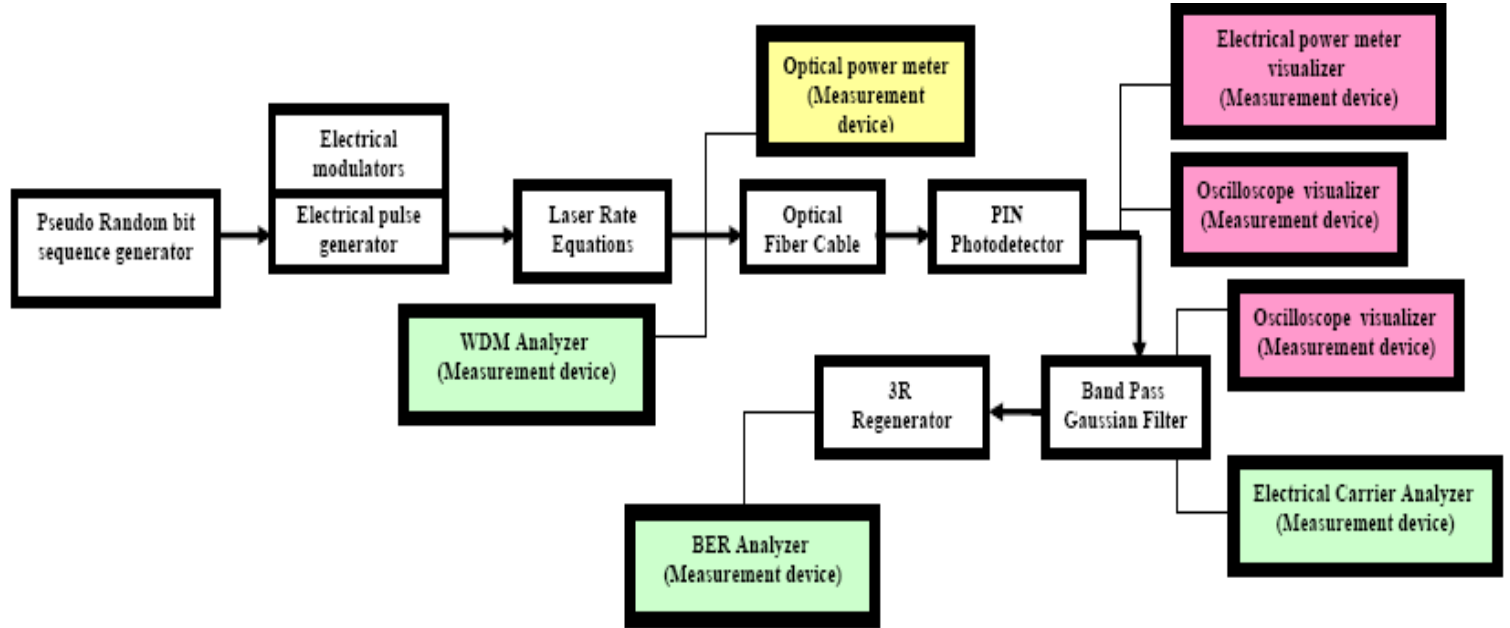

Figure 2. Second proposed simulation model (direct modulation)

\section{PERFORMANCE ANALYSIS WITH DISCUSSIONS}

We have simulated the direct/external modulation laser rate equation systems for the management of relative intensity noise (RIN) by the control of bias current and modulation peak current. Different electrical signal pulse generators and modulators are used in order to measure the signal power level with respect to noise power level. Optical signal to noise ratio after optical fiber cable channel, max. Q-factor. Min. BER, signal to noise ratio after receiver side, signal amplitude and power are also measured under the controlling parameters in Table 1. The important performance parameter for direct/external modulation systems are measured at the optimization stage of laser rate equations by setting the bias current and modulation peak current at the values of $\left(\mathrm{I}_{\text {bias }}=100 \mathrm{~mA}, \mathrm{I}_{\mathrm{m}}=20 \mathrm{~mA}\right)$.

Table 1. List of variables used in the proposed model [7-10].

\begin{tabular}{|c|c|c|}
\hline Components & Parameters & Values/units \\
\hline \multirow[t]{5}{*}{ Laser rate equations } & Frequency & $193.1 \mathrm{THz}$ \\
\hline & Power at bias current & $-30 \mathrm{dBm}$ \\
\hline & Modulation Peak current $\left(\mathrm{I}_{\mathrm{m}}\right)$ & $20 \mathrm{~mA}-100 \mathrm{~mA}$ \\
\hline & Threshold current & $33.45 \mathrm{~mA}$ \\
\hline & Threshold power & $0.035 \mathrm{~mW}$ \\
\hline \multicolumn{3}{|l|}{$\begin{array}{l}\text { PAM modulator, QAM modulator, PSK modulator, DPSK modulator, FSK } \\
\text { modulator, CPFSK modulator, OQPSK modulator, MSK modulator }\end{array}$} \\
\hline & Frequency & $500 \mathrm{MHz}$ \\
\hline RZ pulse, NRZ pulse, Gaussian pulse, Triangle pulse, Impulse, Sine, and & Amplitude & 1 a.u. \\
\hline \multirow[t]{2}{*}{ Raised cosine pulse generators } & Bias & 0 a.u. \\
\hline & Width & $0.5 \mathrm{bit}$ \\
\hline \multirow{4}{*}{ Optical fiber cable } & Attenuation at $1550 \mathrm{~nm}$ & $0.2 \mathrm{~dB} / \mathrm{Km}$ \\
\hline & Dispersion at $1550 \mathrm{~nm}$ & $16.75 \mathrm{ps} / \mathrm{nm} / \mathrm{Km}$ \\
\hline & Dispersion slope at $1550 \mathrm{~nm}$ & $0.075 \mathrm{ps} / \mathrm{nm}^{2} / \mathrm{Km}$ \\
\hline & Effective area at $1550 \mathrm{~nm}$ & $80 \mu \mathrm{m}^{2}$ \\
\hline \multirow[t]{2}{*}{ PIN photodetector } & Dark current & $0 \mathrm{nA}$ \\
\hline & Responsivity & $1 \mathrm{~A} / \mathrm{W}$ \\
\hline \multirow[t]{5}{*}{ Band Pass Gaussian Filter } & Frequency & $500 \mathrm{MHz}$ \\
\hline & Bandwidth & $100 \mathrm{MHz}$ \\
\hline & Insertion loss & $0 \mathrm{~dB}$ \\
\hline & Depth & $100 \mathrm{~dB}$ \\
\hline & Order & 1 \\
\hline Optical attenuator & Attenuation & $27 \mathrm{~dB}$ \\
\hline
\end{tabular}


The results are approved as clarified in Figure. 3-14 based on variables in Table 1. Figure 3 covered the max Q-factor values for different electrical signal pulse generators at the transmitter side based previous model and first proposed model with external modulation. It is observed that raised cosine pulse generator gives better max. Q-factor than other electrical signal generators at the optimization current stage of laser rate equations. Figures 4 and 5 have presented the max Q-factor and min BER values by using eye diagram analyzer with raised cosine pulse generator. Where the max. Q-factor value reaches to 10.53 and min BER value reaches to $9.45 \times 10^{-26}$.

Figure 6 outlines the electrical signal power variations after optical receiver against different electrical signal pulse generators for both previous and second proposed models (direct modulation) at the optimization values of bias current $(100 \mathrm{~mA})$, and modulation peak current $(20 \mathrm{~mA})$. NRZ pulse signal generator presented better performance in maximum electric signal power than other pulse generators whose it value reaches to 485.76. Enhancement percentage ratio in electrical signal power by using NRZ pulse generator is reached to $60.78 \%$ in compared to previous model. In addition to optical signal power after optical fiber cable in relation to both modulation peak current and bias current based laser rate equations (second proposed model) is shown in Figure. 7. It is found that by increasing bias current and modulation peak current parameters of laser rate equations, this results in increasing optical signal power at optical fiber cable channel.

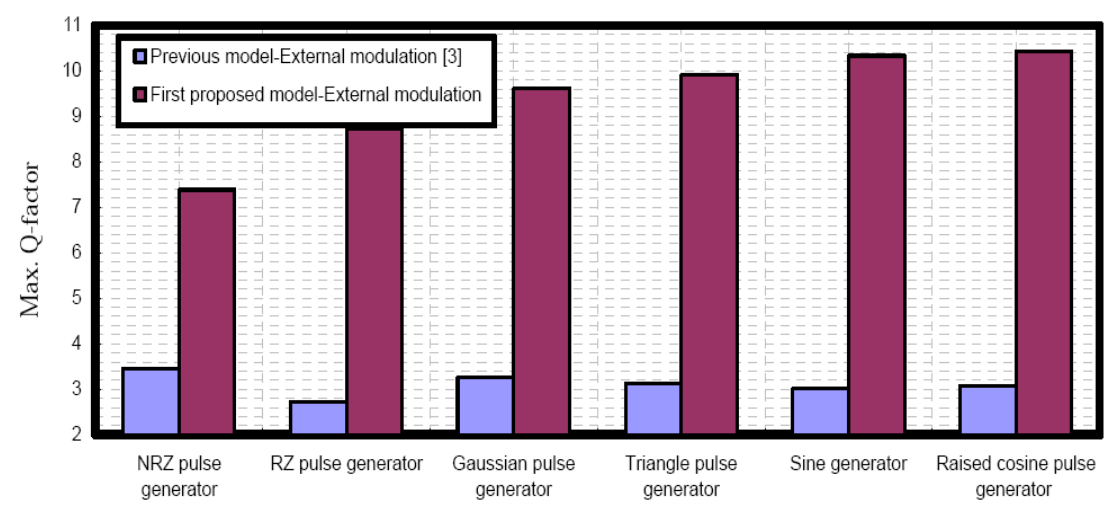

Figure 3. Max Q-factor with different electrical signal pulse generators for both previous and first proposed model with external modulation at the optimization values

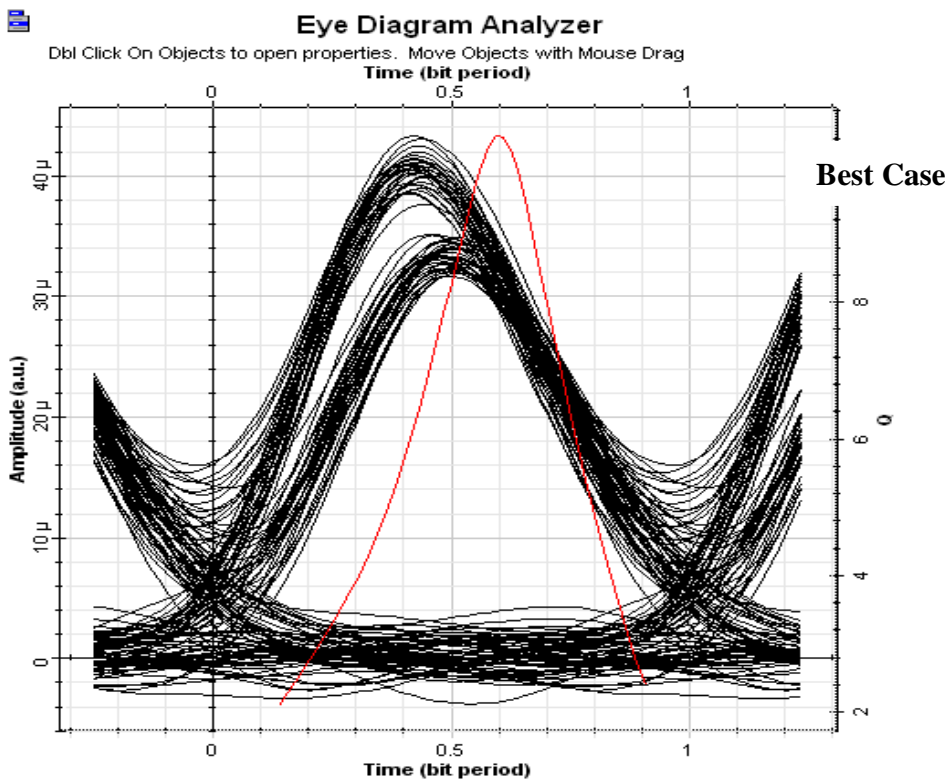

Figure 4. Maximum Q- factor with Raised cosine pulse generator based external modulation using eye diagram analyzer at the optimization stage (First proposed model) 


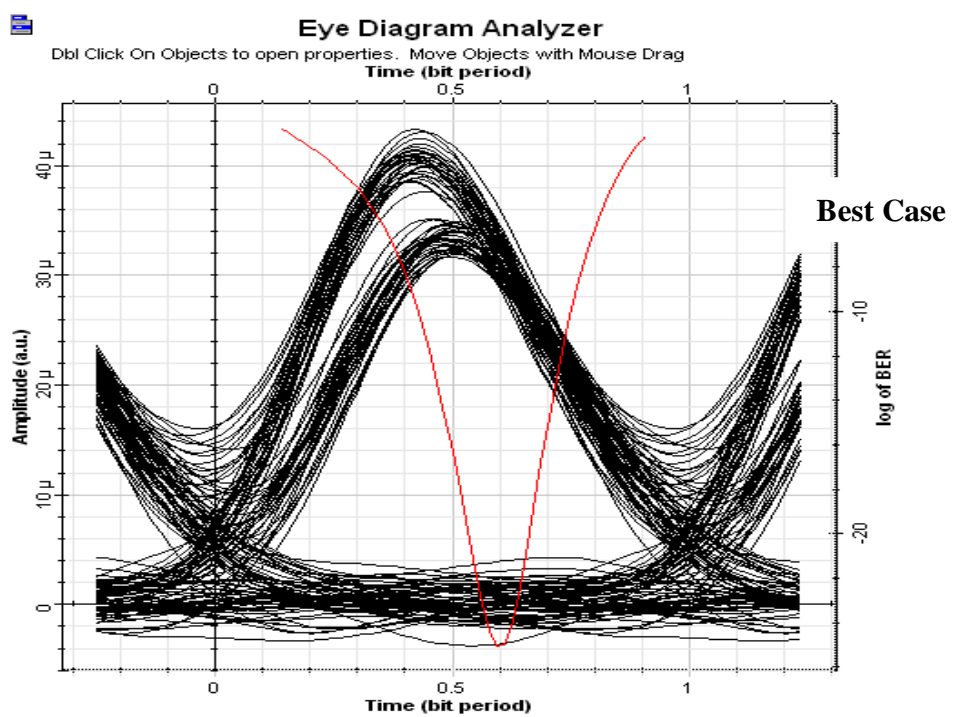

Figure 5. Minimum BER with Raised cosine pulse generator based external modulation using eye diagram analyzer at the optimization stage (First proposed model)

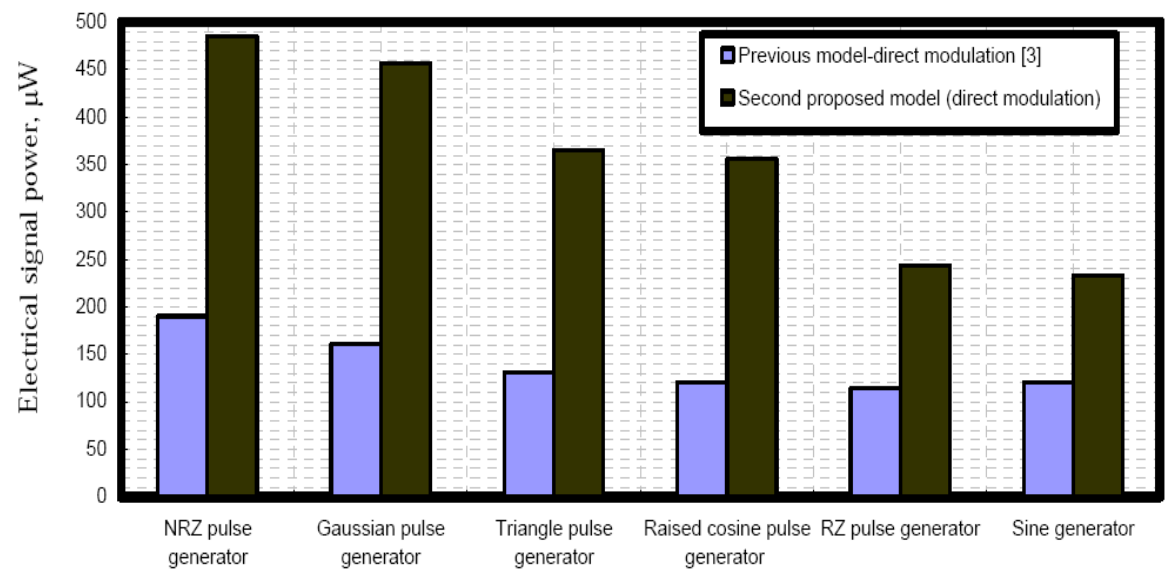

Figure 6. Electrical signal power variations after optical receiver versus different electrical signal pulse generators for both previous and second proposed models (direct modulation) at the optimization values

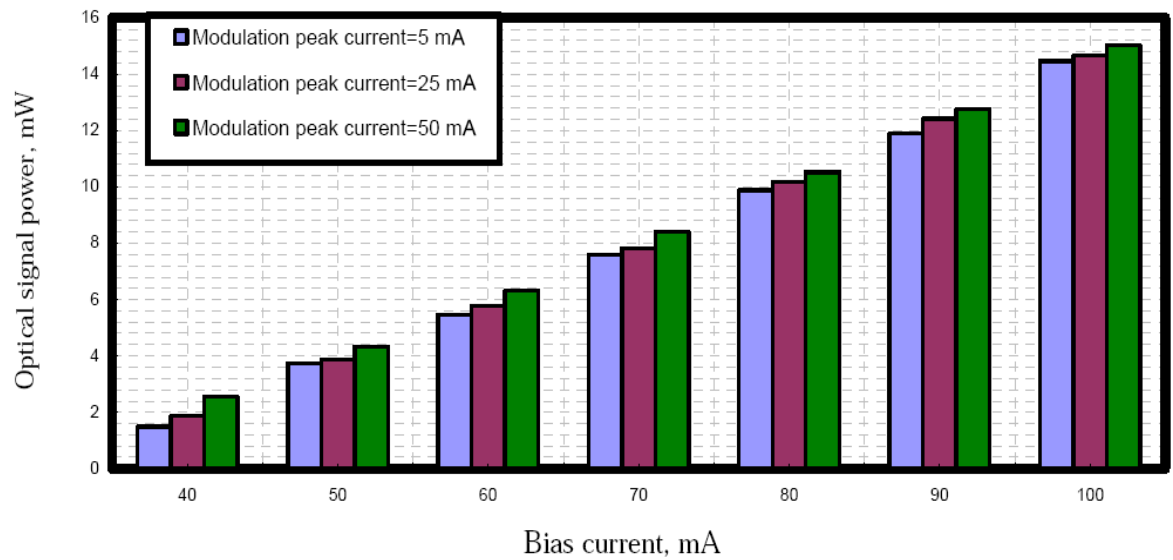

Figure 7. Optical signal power after optical fiber cable in relation to both modulation peak current and bias current based laser rate equations (Second proposed model) 
Figure 8 shows the relation between optical signal to noise ratio after optical fiber cable channel and modulation peak, bias current variations. It is clear that by increasing modulation peak current, this leads to decrease in optical signal to noise ratio for low bias current values $(50 \mathrm{~mA}, 100 \mathrm{~mA})$ but for bias current at a value of $150 \mathrm{~mA}$, the relation is inversely condition. So we recommend for the choice bias current with a value of $100 \mathrm{~mA}$ at the optimization stage. Figure. 9 covered the signal to noise ratio after band pass Gaussian filter (BPGF) in relation to both modulation peak current and bias current based laser rate equations by using second proposed model. Signal to noise ratio after band pass Gaussian filter is enhanced with maximum modulation peak current of $50 \mathrm{~mA}$ at a constant bias current. Signal to noise ratio is enhanced also with increasing bias current expect for modulation peak current value of $5 \mathrm{~mA}$, the relation is inversely condition.

Figures 10 and 11 show the variation of maximum signal amplitude after PIN photodetector, maximum signal amplitude after BPGF against the variations of both modulation peak current and bias current based laser rate equations by using second proposed model. It is clear that the increase in bias current, this results in increasing of maximum signal amplitude after PIN photodetector at constant modulation peak current. It is preferred to choose minimum modulation peak current value of $50 \mathrm{~mA}$ in this case in order to achieve maximum signal amplitude after receiver side. Figure 12 outlines the variation of electrical signal power after PIN photodetector against the variations of both modulation peak current and bias current based laser rate equations by using second proposed model. It is observed that the best choice of bias current value at $100 \mathrm{~mA}$ and modulation peak current of $50 \mathrm{~mA}$ in order to achieve maximum electrical signal power at receiver side. But we recommend to choose modulation peak current less than $50 \mathrm{~mA}$, because the higher modulation peak current, the higher noise power is presented. Figures 13 and 14 covered the optical signal/noise ratio at optical fiber cable and signal/noise ratio after band pass Gaussian filter in relation to different electrical signal pulse generators/electrical signal modulators integrated with laser rate equations at the optimization values by using second proposed model. Both optical signal/noise ratio at fiber cable channel and signal/noise ratio are enhanced better percentage performance by using electrical signal modulators than electrical signal modulators by about $15.88 \%$.

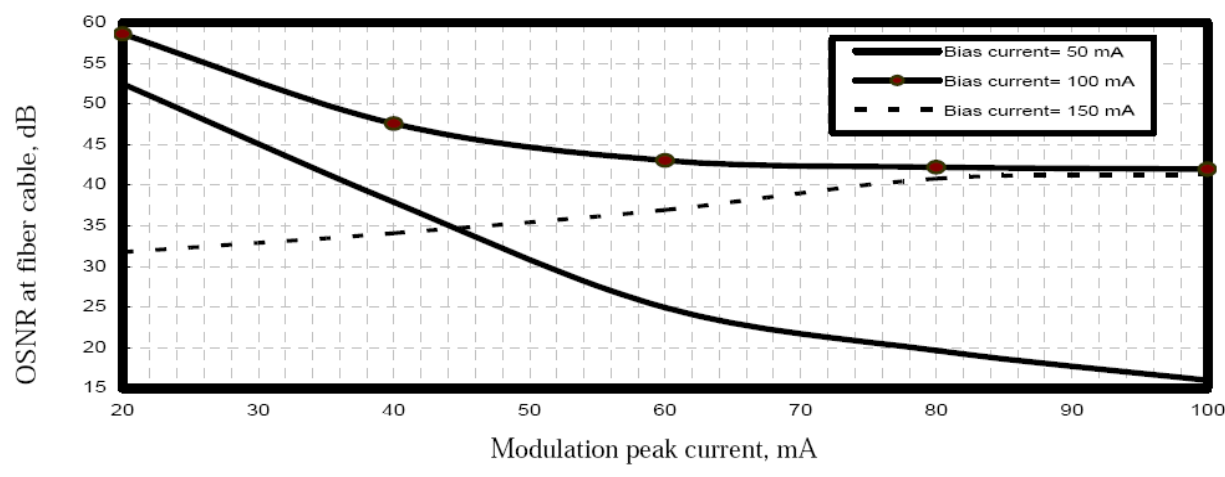

Figure 8. Variations of optical signal to noise ratio at optical fiber cable against variations of both bias current and modulation peak current based laser rate equations (Second proposed model)

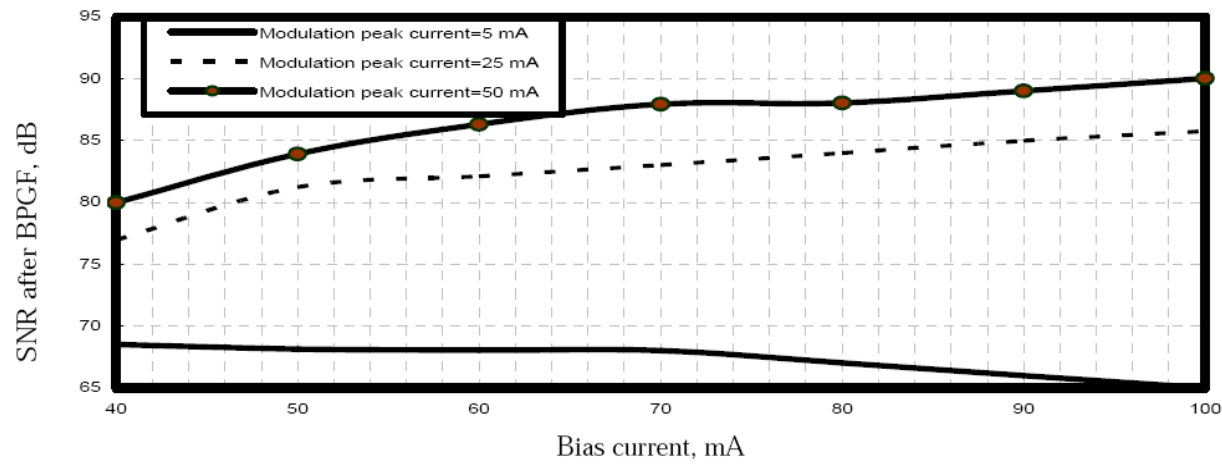

Figure 9. Signal to noise ratio after band pass Gaussian filter (BPGF) in relation to both modulation peak current and bias current based laser rate equations (Second proposed model) 


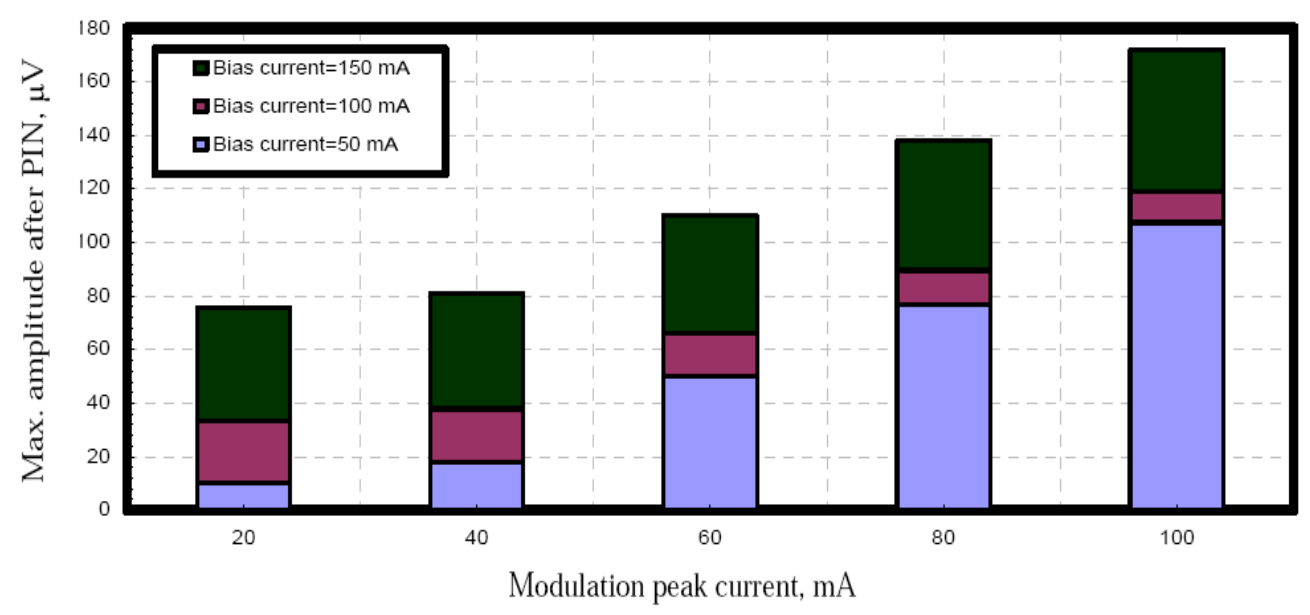

Figure 10. Variation of maximum signal amplitude after PIN photodetector versus the variations of both modulation peak current and bias current based laser rate equations (Second proposed model)

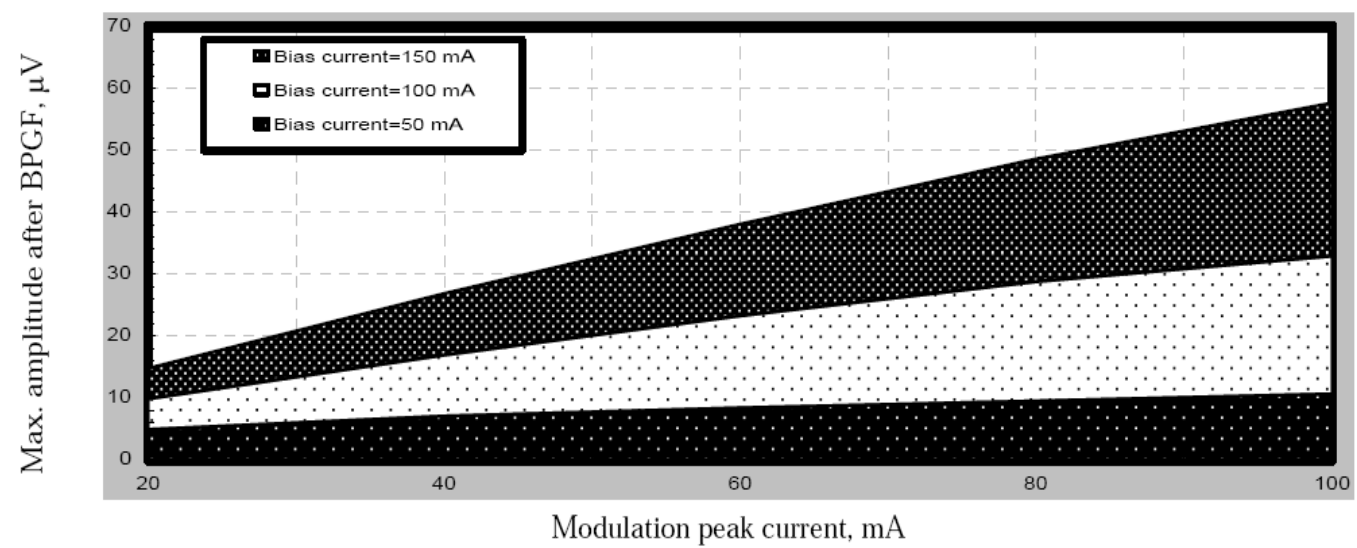

Figure 11. Variation of maximum signal amplitude after BPGF versus the variations of both modulation peak current and bias current based laser rate equations (Second proposed model)

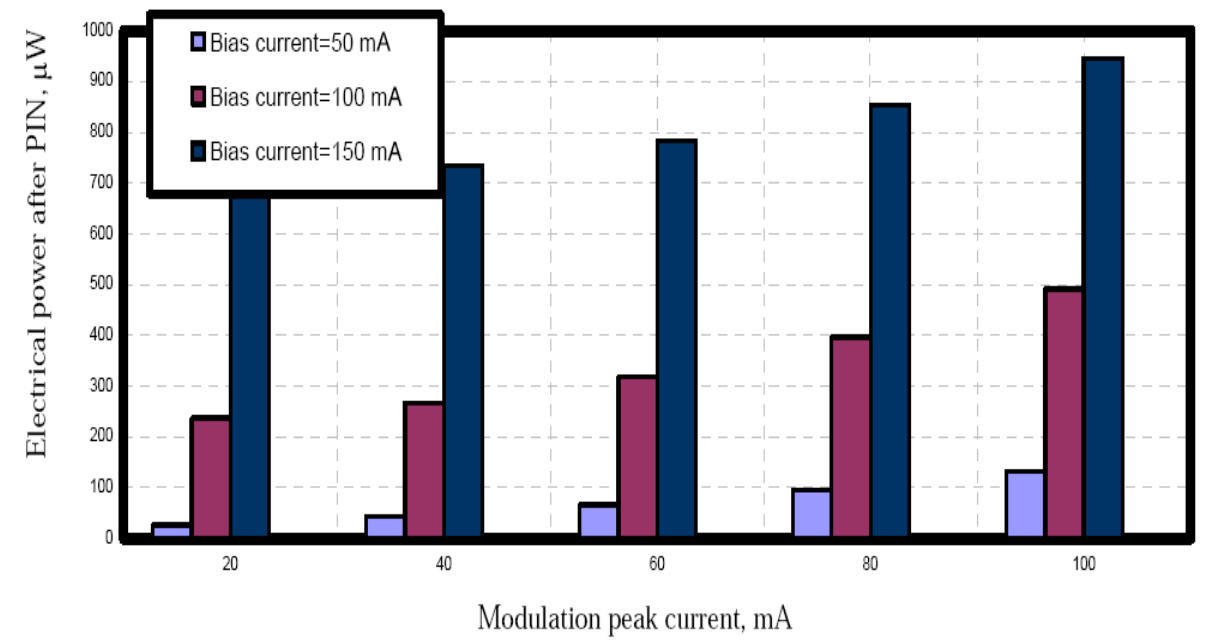

Figure 12. Variation of electrical signal power after PIN photodetector versus the variations of both modulation peak current and bias current based laser rate equations (Second proposed model) 


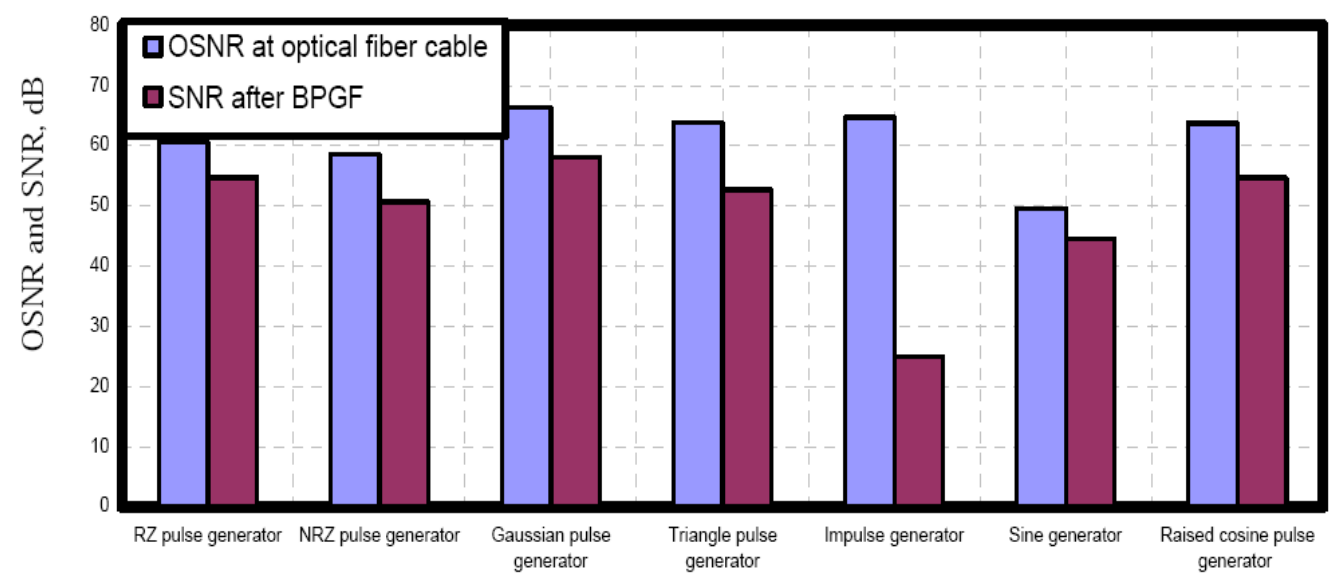

Figure 13. Optical signal/noise ratio at optical fiber cable and signal/noise ratio after band pass Gaussian filter in relation to different electrical signal pulse generators integrated with laser rate equations at the optimization values

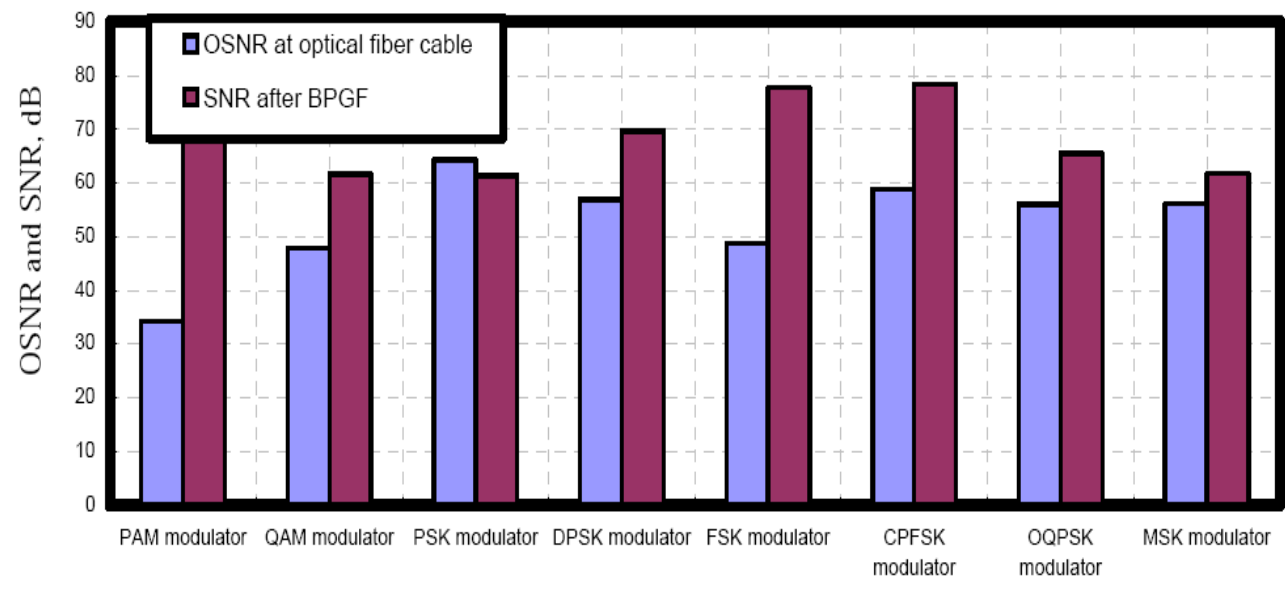

Figure 14. Variations of optical signal to noise ratio at optical fiber cable and signal to noise ratio after band pass Gaussian filter against different electrical modulators integrated with laser rate equations at the optimization values

\section{CONCLUSION}

We have modeled and developed two proposed direct/external modulation laser systems for the key management of relative intensity noise control for the bit rates up to $25 \mathrm{~Gb} / \mathrm{s}$. The laser rate equations operating parameters are optimized through bias current of $100 \mathrm{~mA}$ and modulation peak current of $20 \mathrm{~mA}$. The first proposed model (external modulation) has presented better max. Q-factor and min. BER than previous model (external modulation) at the same simulation parameters for different signal pulse generators. Enhancement percentage ratio in max. Q-factor and min. BER values ranges from $53.25 \%$ to $71.63 \%$ by using first proposed model over the previous model. In addition to electrical signal power is enhanced by using second proposed model (direct modulation) with the previous model. Enhancement percentage ratio in electrical signal power ranges from $48.66 \%$ to $68.88 \%$ by using second proposed model over the previous model. Raised cosine pulse generator is employed efficiently in both proposed models for the percentage enhancement ratio of max. Q-factor, min. BER, and electrical signal power over the previous models (either direct or external modulation). OSNR at optical fiber cable and SNR at optical receiver are enhanced by using electrical modulators (external modulation) than pulse generators (direct modulation) at the optimization case. It is evident that noise power reduction percentage ratio is reached to $37.34 \%$ by using raised cosine pulse generator integrated with optical modulator (external modulation). 


\section{REFERENCES}

[1] A. Mahmoud, et al., "Modeling and Simulation of Dynamics and Noise of Semiconductor Lasers under NTSC Modulation for Use in the CATV Technology," Benisuef University Journal of Basic and Applied Sciences, Elsevier Publisher, vol. 4, pp. 99-108, 2015.

[2] A. Mahmoud, et al., "Optimum Parameters Controlling Distortion and Noise of Semiconductor Laser Under Analog Multichannel Modulation,” Pramana - J. Phys. vol. 9, no. 5, p. 60, 2018. https://doi.org/10.1007/s12043018-1552-y.

[3] T. M. Akinmoladun, et al., "Comparative Study of Chirp in Direct and External Optical Modulation," IRACSTInternational Journal of Computer Science and Information Technology \& Security (IJCSITS), vol. 7, no. 2, pp. 3338, 2017.

[4] S. Pradhan, et al., "Semiconductor Laser Modulation Response by using Optisystem," International Journal of Engineering \& Technology, vol. 3, no. 5, pp. 113-116, 2016.

[5] A. S. Karar, "Digital signal processing for directly modulated lasers in optical fiber communications," $P h . D$ Thesis, pp. 1-197, 2013.

[6] W. E. Kassa, "Electrical modeling of semiconductor laser for high rata rate wireless communication," Ph. D Thesis, pp. 1-163, 2015.

[7] OptiSystem Tutorials, "Optical communication system design software version 13," vol. 1, pp. 1-500, 2014. [Online]. Available: content/uploads/2015/03/OptiSystem_Tutorials_Volume_1.pdf.

[8] O. L. Ladouceur, "Breakthroughs in Photonics 2014: Optical Interconnection Networks" IEEE photonic journal, vol. 7, no. 3, pp. 751-756 2015.

[9] Manisha, and V. Malik, "Effect of Crosstalk in Optical Component", International Journal for Research in Applied Science \& Engineering Technology (IJRASET), vol. 3, no. 5, pp. 456-459, 2015.

[10] R. R. Aggarwal et al., "Multistage Interconnection Networks: A transition from Electronic to Optical," Journal of Emerging Technologies in Web Intelligence, vol. 2, no. 2, pp. 142-147, 2010.

[11] N. A. Yunus, and M. Othman "Reliability Evaluation and Routing Integration in Shuffle Exchange Omega Network", Journal of Networks, vol. 9, no. 7, pp. 1732-1737, 2014.

[12] S. C. Chau et al., "Routing and Scheduling for a Novel Optical Multistage Interconnection Networks," Euro-Par 2005 Parallel Processing, Lecture Notes in Computer Science, vol. 3648, no. 9, pp. 984-993, 2005.

[13] C. Qiao, and R. Melhem, "A Time Domain Approach for Avoiding Crosstalk in Optical Blocking Multistage Interconnection Networks", Journal of Lightwave Technology, vol. 12. no. 10, pp. 1854-1862, 1994.

[14] T. D. Shahida et al., "Fast Zerox algorithm for routing in optical Multistage interconnection networks", IIUM Engineering Journal, vol. 11, no. 1, pp. 28-39, 2010

[15] A.K. Katangur et al., "Analyzing the performance of optical multistage interconnection networks with limited crosstalk", Cluster Computing, vol. 10, no. 7, pp. 241-250, 2007.

[16] F. Abed and M. Othman, "Fast method to find conflicts in optical multistage interconnection networks," International Journal of The Computer Internet and Management, vol. 16, no. 1, pp. 18-25, 2008.

[17] M. Abdullah et al., "An efficient approach for message routing in optical omega network", International Journal of The Computer Internet and Management, vol. 14, no. 1, pp. 50-60, 2006.

[18] M. A. Al-Shabi, "ZeroX Algorithms with Free crosstalk in Optical Multistage Interconnection Network" International Journal of Advanced Computer Science and Applications, vol. 4, no. 2, pp.156-160, 2013.

[19] Ramniwas, and L. Lodha "Reduce the Cross Talk in Omega Network by Using Windowing Techniques", International Journal on Recent and Innovation Trends in Computing and Communication, vol. 4, no. 6, pp. 54-57, 2016.

[20] U. Choudhury, and A. Sangiovanni-Vincentelli, "Constraint-Based Channel Routing for Analog and Mixed Analog/Digital Circuits," IEEE Transactions on Computer-Aided Design of Integrated Circuits and Systems, vol. 12, no. 4, pp. 497-510, 1993.

[21] Kawsar Ahmed, et al., "Design of D-shaped elliptical core photonic crystal fiber for blood plasma cell sensing application," Results in Physics, vol. 12, pp. 2021-2025, 2019.

[22] P. Saxena, and C.L. Liu, "A Post processing Algorithm for Crosstalk-Driven Wire Perturbation," IEEE Transactions on Computer-Aided Design of Integrated Circuits and Systems, vol. 19, no. 6, pp. 691-702, 2000.

[23] T. Gao, and C.L. Liu, "Minimum Crosstalk Channel Routing," IEEE Transactions on Computer-Aided Design, vol. 15 , no. 5 , pp. 465-474, 1996.

[24] H. Zhou, and D.F. Wong, "An Optimal Algorithm for River Routing with Crosstalk Constraints," Proceedings of the International Conference on Computer-Aided Design, pp. 310-315, Nov. 1996.Ahmed Nabih Zaki Rashed, et al., "Performance enhancement of overall LEO/MEO intersatellite optical wireless communication systems," International Journal of Satellite Communications and Networking, vol. 38, no. 1, pp. 31-40, Jan./Feb. 2020. DOI: 10.1002/sat.1306

[25] K. Jhang et al., "COP: A Crosstalk Optimizer for Gridded Channel Routing", IEEE Transactions on ComputerAided Design of Integrated Circuits and Systems, vol. 15, no. 4, pp. 424-429, 1996.

[26] M. Moudia, M. Othman, "Greedy Zero Algorithms for Conflict-Free Scheduling in Low Stage Interconnection Network," Procedia Computer Science, vol. 59, pp. 106-114, 2015.

[27] G. Shanmugam, et al., "heuristic algorithms for vehicle routing problem with stochastic demands," Journal of Computer Science, vol. 7, pp. 533-542, 2011 
[28] M. Al-Shabi, M. Othman, "A new algorithm for routing and scheduling in optical omega network," International Journal of Advanced Computer Science and Applications, vol. 16, no. 1, pp. 26-31, 2008.

[29] R. Bashirov R, T. Karanller, "On path dependent loss and switch crosstalk reduction in optical networks," Information Sciences, vol. 180, no. 6, pp. 1040-1050, 2010.

[30] M. Moudi, M. Othman, "A Cost-Effective Architecture For Optical Multistage Interconnection Network," Journal of Networks, vol. 8, no. 2, pp. 345-350, 2013.

[31] Ahmed Nabih Zaki Rashed, Mohamed Salah F. Tabbour "The engagement of hybrid dispersion compensation schemes performance signature for ultra wide bandwidth and ultra long haul optical transmission systems," Wireless Personal Communications Journal, Published online 19 August, https://doi.org/10.1007/s11277-01906687-2.

[32] Ahmed Nabih Zaki Rashed, et al., "The effect of using different materials on erbium-doped fiber amplifiers for indoor applications," Results in Physics, vol. 15, p. 102650, 2019, https://doi.org/10.1016/j.rinp.2019.102650.

[33] Y. Kaneko, T. Tamanuki, M. Katoh, H. Maekawa, F. Koyama, and K. Iga, "Transverse-mode characteristics of InGaA/GaAs vertical-cavity surface-emitting lasers considering gain offset," Japanese Journal of Applied Physics, vol. 32, pp. 1612-1614, 1993.

[34] E. F. Schubert, N. E. J. Hunt, R. J. Malik, M. Micovic and D. L. Miller, "Temperature and Modulation Characteristics of Resonant-Cavity Light-Emitting Dioded", IEEE Journal of Lightwave Technology, vol 14, pp 1721-1728, 1996.

[35] D. G. Deppe, J. C. Campbell, R. Kuchibhotla, T. J. Rogers and B. G. Streetman, "Optically-coupled MirrorQuantum Well InGaAs-GaAs Light Emitting Diode”, Electronics Letter, vol. 26, p 1665-1666, 1990.

[36] N. Debbar, S. Hong, J. Singh, and P. Bhattacharya, "Coupled GaAs/AlGaAs quantum-well electro absorption modulators for low-electric-field optical modulation", J. Appl. Phys., vol. 65, pp. 383-385, 1989.

[37] V. Kumar, and K. Singh, "Model for calculating the refractive index of different materials", Indian journal of pure and applied physics, Vol. 48, No. 8, pp. 571-574, Aug. 2010.IS Amiri, et al., "High-speed transmission circuits signaling in optical communication systems," Journal of Optical Communications, Published Online: 9 Nov. 2019. https://doi.org/10.1515/joc-2019-0197.

[38] IS Amiri, et al., "Technical Specifications of the Submarine Fiber Optic Channel Bandwidth/Capacity in Optical Fiber Transmission Systems," Journal of Optical Communications, Published Online: 9 Nov. 2019. https://doi.org/10.1515/joc-2019-0226. 